2015-02

\title{
Buckling analysis of partially protected cold-formed steel channel-section columns at elevated temperatures
}

Cheng, Shanshan

http://hdl.handle.net/10026.1/10964

10.1016/j.firesaf.2015.02.010

Fire Safety Journal

Elsevier BV

All content in PEARL is protected by copyright law. Author manuscripts are made available in accordance with publisher policies. Please cite only the published version using the details provided on the item record or document. In the absence of an open licence (e.g. Creative Commons), permissions for further reuse of content should be sought from the publisher or author. 
The paper has been published on:

Fire Safety Journal, Volume 72, Feb 2015, 7-15.

Buckling analysis of partially protected cold-formed steel channel-section columns at elevated temperatures

Shanshan Cheng, Long-yuan Li, Boksun Kim

School of Marine Science and Engineering, University of Plymouth, Plymouth, UK

Fax: +44 (0)1752586101; email address: shanshan.cheng@plymouth.ac.uk

\begin{abstract}
This paper presents a numerical investigation on the buckling behaviour of plasterboard protected CFS channel-section columns subjected to axial loading when exposed to fire on its one side. The work involves temperature-dependent pre-buckling stress analysis and buckling analysis. Two non-uniform temperature distributions with linear and nonlinear temperatures along the web and constant temperatures in flanges and lips have been included in this paper. Bernoulli beam theory has been used in the pre-buckling stress analysis with considering the effects of temperature on strain and mechanical properties. The buckling analysis is performed using combined finite strip analysis and classical Fourier series solutions, in which the mechanical properties are considered to be temperature dependent. The results show that the temperature distributions have a significant influence on both the pre-buckling stress distribution and slenderness of CFS column members. It is also found that the thermal bowing effect improves the buckling performance of intermediate CFS members, but deteriorates that of long CFS members. This paper gives a better understanding of the effect of non-uniform temperatures on the buckling behaviour of CFS columns, and further extends the potential application of the finite strip method to the buckling analysis of CFS members at elevated temperatures.
\end{abstract}

Keywords: Cold-formed steel; buckling; fire; temperature; channel; columns.

\title{
1. Introduction
}

Thin-walled, cold-formed steel (CFS) members have been increasingly used as load-bearing components in low- and mid-rise buildings due to its advantages of high strength-to-weight ratio, ease of fabrication, and the flexibility of sectional profiles. However, the features such as thin thickness, open cross-section and great flexural rigidity difference about two crosssectional axes, lead the buckling failure to be the main failure mode of CFS members [1]. When it is exposed to a fire, the rapid temperature rise in a CFS member makes the buckling behaviour even worse [2]. If the temperature distribution in a member is uniform, the buckling behaviour of the member can be analysed based on uniformly reduced material properties. However, if the temperature distribution in a member is not uniform, which usually happens in walls and/or floor panels when CFS members are exposed to fire on one side, the temperature-dependent material properties vary within the member. Together with the thermal bowing effect caused by various thermal expansions on the member, the analysis of structures could be much complicated, the problem of which is not fully addressed in the existing analysis of CFS members.

The buckling resistance of CFS columns at elevated temperatures has been investigated by many researchers. Mechanical properties of CFS have been investigated by Chen and Young [3] and Kankanamge and Mahendran [4], which showed a remarkable difference on the reductions of yield strength and elastic modulus when compared to hot-rolled steels. Feng 
and Wang [5] and Feng et al. [6] conducted experimental and numerical studies on the axial strength of CFS channel columns in wall panel systems exposed to fire on one side. It was found that the non-uniform temperature distributions have significant influence on the fire resistance of CFS wall panels. To better understand the fire performance of CFS wall panel systems, the effect of different insulation materials, insulation methods [7], and various fire protection wall panels [8] on the fire performance of the CFS wall systems has been studied recently.

Based on the available experimental and numerical studies of CFS members at elevated temperatures, many modifications have been proposed to the design methods in order to promote the potential applications of CFS. A modification to the design method recognized in EN1993-1-3 [9] was presented by Feng and Wang [10] to take into account the effect of the reduced mechanical properties and thermal bowing deflection at elevated temperatures. Shahbazian and Wang [11-13] presented a design method based on Direct Strength Method (DSM) to calculate the critical buckling load of CFS columns under high temperatures. Gunalan and Mahendran [14] proposed fire design rules based on the CFS design codes AS/NZS 4600 [15] and NAS [16] at room temperature.

Among all the design methods, DSM [17] has been increasingly adopted as an alternative design method to the Effective Width Method (EWM) due to the advantages of no need for tedious calculation of effective width. However, an accurate calculation of member elastic buckling load is essential when using DSM since the resistance of the member is directly determined by the elastic buckling loads and the squash load. The eigenvalue analysis of the Finite Strip Method (FSM) based software [18] has been widely applied to get the elastic buckling loads. However, since most existing FSM based software are developed under ambient temperature, the effect of thermal expansion on the stress distribution and the shift of neutral axis under non-uniform temperatures are not taken into account in these codes. Although the temperature effect has been considered by Shahbazian and Wang [11] in columns by using an "effective" squash load, this simplified method may not be able to reveal the actual non-uniform stress-distribution on the cross section and the corresponding effect on the buckling performance of CFS members. Furthermore, most existing FSM based codes are only available to the members subjected to uniform longitudinal stresses. Therefore it is not able to reveal the feature of the thermal bending moment which varies along the member length with a maximum value at the middle of the member and a zero value at support ends. The present study is therefore to further explore the application of the FSM to the buckling analysis of CFS members at elevated temperatures by considering both the reduced material properties and non-uniform stress distributions within the cross-section and along the member at elevated temperatures.

\section{Pre-buckling stress analysis}

If the channel section has a uniformly distributed temperature the reduction of material properties is identical everywhere in the section. The cross section will be subjected to uniform compression when the member is subjected to an axial load acted at the centroid of the cross section. However, if the temperature distribution is not uniform the stress distribution on the cross-section would be also not uniform, which is highly dependent on the temperature distribution due to the varying reduction of the mechanical properties within the cross-section and the bending moment caused by thermal bowing effect. 
It is well known that steel has large thermal conductivity coefficient and thus for most cases the temperature in the steel can be approximately treated to be uniform. However, in the CFS sections, if the heat is transferred only from its one side and all of other sides are exposed in a cooling air environment, then the temperature variation within the cross-section will be remarkable owing to the thin thickness of the CFS sections. This happens in the external walls where the inner layer uses CFS sections protected by plasterboard. When there is a room fire the heat is transferred from the plasterboard to the CFS sections. In this case the CFS section is subjected to elevated temperatures on its one side and exposed to ambient on all of other sides (see Fig. 1(a)).

The heat transfer can be analysed using finite element analysis packages such as ANSYS. Since the longitudinal dimension is much greater than the cross-section dimensions, the heat transfer analysis can be carried out by using a 2-dimensional geometric model as shown in Fig. 1(a). A CFS channel section with a web depth of $200 \mathrm{~mm}$, flange width of $75 \mathrm{~mm}$, lip length of $20 \mathrm{~mm}$ and thickness of $2 \mathrm{~mm}$ is analysed, and a single layer of plasterboard with a thickness of $12.5 \mathrm{~mm}$ is used. All material properties such as density, specific heat, and thermal conductivity for CFS and plasterboard are assumed to be temperature-dependent, which are provided in $[19,20]$. The initial temperatures in both the CFS and plasterboard are assumed to be ambient. The combined convection and radiation boundary conditions are applied to all boundaries. However, the environmental temperatures surrounding the section are different. For the fire exposed surface (one side of the plasterboard) the environmental temperature is the fire temperature, which is calculated based on the standard fire curve; whereas for the fire unexposed surfaces the environmental temperature is the room temperature $\left(20^{\circ} \mathrm{C}\right)$. The convective coefficients employed in the fire exposed and fire unexposed surfaces are 25 and $9 \mathrm{~W} / \mathrm{m}^{2} \mathrm{~K}$, respectively.

Figure 1(b) shows the temperature distribution obtained from the finite element analysis. It can be seen from the figure that the temperature exhibits highly nonlinear on the flange, lip and web near to the plasterboard exposed to the fire. In order to examine the effect of nonlinear distribution of temperature on the buckling behaviour of the CFS section members, two simplified temperature distributions shown in Fig. 1(c) and (d) are proposed for the prebuckling stress analysis. In Fig. 1(c) the temperature is assumed to be constants in flanges. Along the web the temperature decreases parabolically from fire exposed side to the geometrical centroid axis; afterwards the temperature is assumed to be linear till the fire unexposed side. In Fig. 1(d) the temperature is also assumed to be constants in flanges, but in the web and lips it is assumed to be linearly varied. The temperature on the flange near to the fire exposed side is taken as the highest temperature in that flange, while the temperature on the other flange is taken as the room temperature. Note that the temperature distribution in Fig. 1(c) is closer to Fig. 1(b) than that in Fig. 1(d).

Let $o$ be the centroid of the channel-section, $x$ be the longitudinal axis, $y$ and $z$ be the two geometrically principal axes of the cross-section, respectively. By using Bernoulli beam's assumption, the axial strain at any coordinate point of the cross-section can be expressed as the sum of a membrane strain and two bending strains as follows,

$$
\varepsilon(y, z)=\varepsilon_{o}+y \kappa_{x y}+z \kappa_{x z}
$$

where $\varepsilon_{o}$ is the membrane strain, $\kappa_{x y}$ and $\kappa_{x z}$ are the curvatures of the beam in $x y$ - and $x z-$ planes, respectively. On the other hand, the total axial strain can also be decomposed in terms of the components generated by individual actions, 
$\varepsilon(y, z)=\frac{\sigma_{s}}{E(T)}+\varepsilon_{t h}(T)$

where $\sigma_{s}$ is the axial stress, $E$ is the temperature-dependent Young's modulus, and $\varepsilon_{t h}$ is the thermal strain. Solve $\sigma_{s}$ from Eqs. (1) and (2), yielding,

$$
\sigma_{s}=E\left(\varepsilon_{o}+y \kappa_{x y}+z \kappa_{x z}-\varepsilon_{t h}\right)
$$

The resultant axial load and moments about two principal axes on the cross-section requires the following equilibrium equations,

$$
\begin{aligned}
N_{x}= & \int_{A} \sigma d A=\int_{A} E\left(\varepsilon_{o}+y \kappa_{x y}+z \kappa_{x z}-\varepsilon_{t h}\right) d A \\
& =\varepsilon_{o} \int_{A} E d A+\kappa_{x y} \int_{A} y E d A+\kappa_{x z} \int_{A} z E d A-\int_{A} E \varepsilon_{t h} d A=0 \\
M_{z}= & \int_{A} y \sigma d A=\int_{A} y E\left(\varepsilon_{o}+y \kappa_{x y}+z \kappa_{x z}-\varepsilon_{t h}\right) d A \\
& =\varepsilon_{o} \int_{A} y E d A+\kappa_{x y} \int_{A} y^{2} E d A+\kappa_{x z} \int_{A} y z E d A-\int_{A} y E \varepsilon_{t h} d A \\
M_{y}= & \int_{A} z \sigma d A=\int_{A} z E\left(\varepsilon_{o}+y \kappa_{x y}+z \kappa_{x z}-\varepsilon_{t h}\right) d A \\
& =\varepsilon_{o} \int_{A} z E d A+\kappa_{x y} \int_{A} y z E d A+\kappa_{x z} \int_{A} z^{2} E d A-\int_{A} z E \varepsilon_{t h} d A
\end{aligned}
$$

where $N_{x}$ is the axial load, $M_{y}$ and $M_{z}$ are the bending moments about $y$ - and $z$-axes, respectively. Since the member is subjected to an axial load only, $M_{y}$ and $M_{z}$ are equal to zero under uniform temperatures, but may not be zero under non-uniform temperatures. Due to thermal expansions on the cross-section, the member would have a bow towards the high temperature side. The axial load $N_{x}$ applied at the centroid at the ends of the column then would behave like an eccentric load along the member, and a thermal bending moment would be brought to the member. In the two temperature distributions shown in Fig. 1 (c) and (d), the temperature is constant along z- axis, therefore the bending moment $M_{y}$ is equal to zero under this temperature. The thermal bowing is assumed to vary parabolically along the member with a maximum value at the middle [21] and zero value at two ends. $M_{z}$ is thus taken as the product of the axial load and the thermal deflection, as shown in Eqs. (7) and (8),

$$
\begin{aligned}
& M_{z}=\frac{4\left(L \cdot x-x^{2}\right)}{L^{2}} \cdot M_{\text {max }} \\
& M_{\text {max }}=N_{x} \cdot \frac{\alpha L^{2} \Delta T}{8 d}
\end{aligned}
$$

where $L$ is the member length, $\alpha$ is the thermal expansion coefficient of the CFS, $\Delta T$ is the temperature difference between the two flanges, and $d$ is the depth of the cross-section.

Eqs. (4)-(6) can be used to determine the membrane strain $\left(\varepsilon_{o}\right)$ and bending curvatures $\left(\kappa_{x y}\right.$ and $\kappa_{x z}$ ) of the member, which are then substituted into Eq. (3) to obtain the axial stress $\sigma_{s}$.

Figs. 2 and 3 show the stress distributions of a CFS channel-section column, with a member length of $6 \mathrm{~m}$, under both linear and nonlinear temperature distributions as proposed in Fig. 1. The axial load is assumed as the yield strength at ambient temperature $\left(N_{x}=f_{y, T 0} \cdot A\right.$ where $f_{y, T 0}$ is the yield stress at ambient temperature and $A$ is the gross cross-sectional area of the column) and the compressive stress is taken to be positive and tensile stress is negative. Note that the thermal bending moment is dependent on the member length and thus the change in the column length will lead to a different stress distribution along the member. 
Figs. 2(a)-2(c) show the stress distributions at the end of the column under three linear temperature distributions. Since the thermal bending at the end is zero, Figs. 2(a)-2(c) reveal the pure effect of the shift of centroid caused by the variation of elastic modulus within the cross-section. Through the figures it can be seen that the member is under pure compression, and the stress distribution tends to be nonlinear along the web, but linear along the flanges and lips, with the highest stress at the junction of the fire unexposed flange and fire unexposed lip, and the lowest stress at the junction of the fire unexposed flange and the web. Figs. 2(d)-2(f) show the stress distributions at the middle section of the column. It can be seen from the figures that the thermal bending moment exhibits a remarkable influence on the stress distribution when they are compared with Figs. 2(a)-2(c). The compressive stress on the fire unexposed side is significantly increased while the compressive stress on the fire exposed side is reduced considerably and turned to tension when the temperature reaches about $300{ }^{\circ} \mathrm{C}$. When the temperature is low (shown in Fig. 2(d)), the highest stress occurs at the junction of the fire unexposed flange and the fire unexposed lip. However the highest stress point changes to the junction of the fire unexposed flange and the web when the temperature exceeds $300^{\circ} \mathrm{C}$.

Figs. 3(a)-3(c) show the stress distributions at the end of the column under nonlinear temperature distributions. Due to the nonlinearity of the thermal expansion within the crosssection, the mechanical strain shown in Eq. (3) tends to be nonlinear in order that the crosssection remains in the plane. This brought into a tension to the region with a sharp reduction of the high temperature (on the web near the fire exposed flange), which is revealed by a remarkable reduction of the compression on the web near the fire exposed flange. The compression is shifted to other regions. Therefore the highest compressive stress is exhibited higher than that shown in Figs. 2(a)-2(c). When the temperature is less than $300{ }^{\circ} \mathrm{C}$, the highest compression occurs at the junction of the fire exposed flange and the web. However when the fire reaches $600{ }^{\circ} \mathrm{C}$ the highest compressive stress moves to the web since the compression on the fire exposed flange reduces obviously, due to the remarkable reduction of the elastic modulus under high temperature. When taking into account the thermal bending, shown by the stress distributions on the middle section in Figs. 3(d)-3(f), the tension caused by various thermal expansions increased the value of tensile stress on the web and extended the tension region obviously under high temperatures. It is observed that the maximum stress is generally higher in Fig. 3 than that shown in Fig. 2 under the same high temperature, which may lead to earlier material yield under nonlinear temperature distribution; that is a smaller slenderness.

\section{Modified FSM}

Buckling analysis can be completed in a variety of ways. The commonly used methods include the finite element analysis, finite strip analysis, classical Fourier series solutions, and generalized beam theory method [22]. For CFS members subjected to pure compression and/or pure bending, the finite strip analysis is the most effective method [23]. However, if the CFS member is under non-uniform temperatures, one has to combine the finite strip analysis and classical Fourier series solutions [24,25] due to the variation of pre-buckling stresses along the longitudinal axis.

A modified semi-analytical FSM has been developed, which takes into account the reduced material properties together with non-uniform per-buckling stress distributions at elevated temperatures. Note that the additional thermal bending moment varies parabolically along the 
member, while the axial loading remains unchanged. The stress distribution is therefore divided into two parts. One is the stress caused by the axial loading, which does not vary along the length of the member. The other is the stress caused by the thermal bending moment, which varies parabolically from zero at the ends to the maximum value at the middle of the member. For each strip of the cross-section, the local geometric stiffness matrix is calculated based on each stress part and then assembled into the overall local geometric stiffness matrix, as shown in Eq. (9):

$k_{g}=k_{g}^{\prime}+k_{g}^{\prime \prime}$

where $k_{g}^{\prime}$ is the local geometric stiffness matrix caused by axial loading, and $k_{g}^{\prime \prime}$ is the one caused by thermal bending. $k_{g}^{\prime}$ and $k_{g}^{\prime \prime}$ are shown in Eqs. (10) and (11) respectively.

$$
\begin{aligned}
& k_{g}^{\prime}=\int_{0}^{a} \int_{0}^{b}\left(T_{1}-\left(T_{1}-T_{2}\right) \frac{y}{b}\right)\left(\left(\frac{\partial u}{\partial x}\right)^{2}+\left(\frac{\partial v}{\partial x}\right)^{2}+\left(\frac{\partial w}{\partial x}\right)^{2}\right) d x d y \\
& k_{g}^{\prime \prime}=\int_{0}^{a} \int_{0}^{b} f(x)\left(T_{1}^{\prime}-\left(T_{1}^{\prime}-T_{2}^{\prime}\right) \frac{y}{b}\right)\left(\left(\frac{\partial u}{\partial x}\right)^{2}+\left(\frac{\partial v}{\partial x}\right)^{2}+\left(\frac{\partial w}{\partial x}\right)^{2}\right) d x d y
\end{aligned}
$$

Where $T_{1}, T_{2}, T_{1}$ ', and $T_{2}$ ' are the loads on the two edges of the strip caused by axial loading and thermal bending respectively; $f(x)=\frac{4\left(L \cdot x-x^{2}\right)}{L^{2}}$ is the function describing the variation of axial compressive stress.

The local initial stiffness matrix is calculated based on the shape function of the deformation [23], which is independent of the stress. After all local matrices are formed, global stiffness and global geometric stiffness matrices are assembled and the lowest eigenvalue of the generalised eigenvalue matrix equation is solved, from which the critical buckling load is obtained. The details of the finite strip method could be found in [23] and thus is not provided here. Similar matrix equations to those given in $[24,25]$ but with different matrix coefficients, regarding to temperature effects, can be found in [19]. The second differece between the modified FSM and the traditional FSM is that the coefficient matrices of trigonometric functions are coupled between individual wave numbers because of the involvement of $\mathrm{f}(\mathrm{x})$ in Eq.(11) and and thus the lowest eigenvalue is no longer related to the wave number or the half-wavelength.

In the present buckling analysis, the temperature-dependent mechanical properties of CFS, proposed by Kankanamge and Mahendran [4] based on their experimental results, are adopted. The accuracy of the modified FSM has been validated using the finite element analysis (ANSYS), as shown in Table 1. It can be seen from the table that, the modified FSM provides very close results to those given by the FEM, except for three intermediate columns with nonlinearly distributed temperatures where the modified FSM provides slightly larger critical loads than the FEM does. In order to examine the feasibility of the assumption of uniform thermal bending employed in the buckling analysis of CFS columns in fire, Fig. 4 shows the comparison of the buckling loads, obtained from the modified FSM, by using both uniform and parabolic thermal bending moments. It is indicated that using a uniform thermal bending is acceptable for member lengths shorter than $2 \mathrm{~m}$. However, when the member is longer than $2 \mathrm{~m}$, using uniform thermal bending provides either unsafe or over conservative results for both linear and nonlinear temperature distributions. The error increases remarkably 
with increasing member lengths, which concludes that using uniform thermal bending moment may not be reliable for long members. Note that the traditional FSM cannot take account the non-uniform thermal bending, whereas the modified FSM can. Thus the latter is used in the buckling analysis which is shown in the next section.

\section{Buckling analysis}

Fig. 5 shows the dimensionless critical buckling loads $P_{c r} / P_{y, T o}$ (where $P_{c r}$ is the critical buckling load and $P_{y, T o}=f_{y, T o} A$ is the yield load of the CFS column at room temperature) of the CFS channel section column under both linear and nonlinear temperature distributions. In general, the critical buckling load of the CFS column under nonlinear temperature distribution is higher than that under the linear temperature distributions. In order to examine the effect of thermal bowing on the buckling behaviour, buckling analyses with and without considering the thermal bowing are conducted. The buckling loads considering thermal bowing effect are shown by circle markers, while the ones without thermal bowing effect are shown by solid curves. It can be seen from the figure that the thermal bowing has almost no effect on the local buckling of short members but has some positive effect on the local buckling of intermediate members. Also, it can be observed from the figure that, the effect of thermal bowing on the global buckling is heavily dependent on the member length. For members that are not very long the thermal bowing has a positive effect on the global buckling of the member; however, for members that are very long, i.e., high slender members, and with high temperatures the thermal bowing can reduce the critical load of global buckling, as is demonstrated in Fig. 5(c). The reason for this is due to the fact that when the member is short, the buckling happens on the fire exposed side, as shown in Fig. 6. The added thermal bowing reduces the stress value on the buckling region, which consequently improves the buckling performance. However, if the member is long, the buckling happens on the fire unexposed side. The bending caused by thermal bowing weakens the buckling performance accordingly. Furthermore, the buckling load decreases gradually with the rise of temperature when the temperature distribution is linear. However when the temperature distribution is nonlinear within the cross-section, the local buckling load exhibits a slightly increase when the temperature is less than $400{ }^{\circ} \mathrm{C}$, and then reduces considerably later on.

The buckling modes of the column under the linear and nonlinear temperature distributions are shown in Fig. 7. Since the member is predominantly in compression the buckling modes are mainly the local and flexure buckling modes. No distortional buckling mode was found. It can be seen from the figure that the main difference between the two buckling modes is the deformed shape. In the linear temperature distribution the local buckling occurs in the web only; whereas in the nonlinear temperature distribution the local buckling occurs not only in the web but also in the fire exposed flange.

In order to examine whether the material yield has occurred when the column buckles, Fig. 8 re-plots the critical loads of Fig. 5 by using the relative critical buckling load $P_{c r} / P_{y, T}$, where $P_{y, T}$ is the yield/squash load of the CFS column under high temperature. Since it is not recommended to use the inelastic capacity of CFS sections due to the high slenderness of the cross-section, the elastic cross-section limit is applied herein. The relationship between $P_{y, T}$ and $P_{y, T o}$ is shown in Fig. 9. The blue curve exhibits the stress value $\sigma_{y, T o}$ according to Fig. 2(b), while the pink dashed curve shows the yield stress $f_{y, T}$ on the cross-section under the corresponding non-uniform temperature distribution. The area bounded by the blue curve and the axes reveals the axial loading $P_{y, T 0}$ on the cross-section. To make sure the cross-section is 
under elasticity, a reduction factor $\eta$, taken as the minimum ratio of $f_{y, 7} / \sigma_{y, T o}$, is adopted. The yield stress distribution $\sigma_{y, T}=\sigma_{y, T o} * \eta$ is shown as the red curve in Fig. 9, and the yield load $P_{y, T}$ is taken as the area bounded by the red curve and the axes.

It is exhibited in Fig. 8 that the relative buckling load increases with the rise of temperature, due to the fact that $P_{y, T}$ decreases considerably. This indicates that the slenderness of the CFS channel-section column decreases under both non-uniform temperature distributions. However, Figs. 8(a) and 8(b) show considerable difference on the relative buckling load with the rise of temperature. It is exhibited in Fig. 8(a) that the relative local buckling load decreases slightly when $T<300{ }^{\circ} \mathrm{C}$ but increases dramatically afterwards. The reason of the reversion of the slenderness is mainly due to the different reduction rates of the Young's modulus and yield strength of the CFS material in the temperature regions less and greater than $300{ }^{\circ} \mathrm{C}$. Whereas in Fig. 8(b) the relative local buckling load exhibits a dramatic rise even at low temperatures, which may be due to the highly nonlinear stress distributions under nonlinear temperature distributions. Fig. 8 indicates the importance of using an accurate temperature distribution in predicting the buckling behaviour of CFS members in fire.

\section{Conclusions}

A numerical study on the buckling analysis of CFS channel section columns under crosssectional non-uniform temperature distributions has been carried out in this paper. Both linear and nonlinear temperature distributions have been considered and their corresponding effects on the critical buckling load have been discussed. The pre-buckling stress analysis has been completed by using the Bernoulli bending theory considering the effects of temperature on both strain and mechanical properties. The buckling analysis has been performed by using combined finite strip analysis and classical Fourier series solutions, in which the mechanical properties are considered to be temperature dependent. From the results obtained the following conclusions can be drawn:

- The pre-buckling stress distributions are significantly affected by the temperature distributions on the cross-section. With the same axial load and maximum temperature on the cross-section a nonlinear temperature distribution causes a much higher value of the maximum compressive stress, which may lead to an earlier material yield.

- Thermal bowing influences the critical buckling load of intermediate and long CFS columns significantly but has almost no effect on the buckling behaviour of short members. The critical buckling load of intermediate members would be improved when taking into account the thermal bowing effect. However when the member is very slender, the buckling performance would be deteriorated considerably.

- The temperature distribution has a remarkable influence on the critical buckling load and the slenderness of the CFS columns. The use of accurate temperature distributions is of great importance for accurately predicting the buckling behaviour of CFS columns.

Acknowledgement - The first author wishes to acknowledge the financial support received from the Chinese Scholarship Council for her $\mathrm{PhD}$ study being carried out at Plymouth University.

\section{References}


[1] L.Y. Li, X.T. Chu, Cold-formed steel sections, in: Structural design of steelwork, 2nd ed., Elsevier, Amsterdam, 2008, pp. 413-457.

[2] J.A. Purkiss, L.Y. Li, Fire safety engineering design of structures, 3rd ed., CRC Press, UK, 2013.

[3] J. Chen, B. Young, Experimental investigation of cold-formed steel material at elevated temperatures, Thin-Walled Structures 45 (2007) 96-110.

[4] N.D. Kankanamge, M. Mahendran, Mechanical properties of cold-formed steels at elevated temperatures, Thin-Walled Structures 49 (2011) 26-44.

[5] M. Feng, Y.C. Wang, An experimental study of loaded full-scale cold-formed thin-walled steel structural panels under fire conditions, Fire Safety Journal 40 (2005) 43-63.

[6] M. Feng, Y.C. Wang, J.M. Davies, Axial strength of cold-formed thin-walled steel channels under non-uniform temperatures in fire, Fire Safety Journal 38 (2003) 679-707.

[7] S. Gunalan, P. Kolarkar, M. Mahendran, Experimental study of load bearing cold-formed steel wall systems under fire conditions, Thin-Walled Structures 65 (2013) 72-92.

[8] W. Chen, J. Ye, Y. Bai, et al., Full-scale fire experiments on load-bearing cold-formed steel walls lined with different panels, Journal of Constructional Steel Research 79 (2012) 242-254.

[9] EN1993-1-3, Eurocode 3: Design of steel structures, European Commission for Standardisation, Brussels, 2001.

[10] M. Feng, Y.C. Wang, An analysis of the structural behaviour of axially loaded full-scale cold-formed thin-walled steel structural panels tested under fire conditions, Thin-Walled Structures 43 (2005) 291-332.

[11] A. Shahbazian, Y.C. Wang, Calculating the global buckling resistance of thin-walled steel members with uniform and non-uniform elevated temperatures under axial compression, Thin-Walled Structures 49 (2011) 1415-1428.

[12] A. Shahbazian, Y.C. Wang, Application of the Direct Strength Method to local buckling resistance of thin-walled steel members with non-uniform elevated temperatures under axial compression, Thin-Walled Structures 49 (2011) 1573-1583.

[13] A. Shahbazian, Y.C. Wang, Direct Strength Method for calculating distortional buckling capacity of cold-formed thin-walled steel columns with uniform and non-uniform elevated temperatures, Thin-Walled Structures 53 (2012) 188-199.

[14] S. Gunalan, M. Mahendran, Development of improved fire design rules for cold-formed steel wall systems, Journal of Constructional Steel Research 88 (2013) 339-362.

[15] Standards Australia (SA), AS/NZS 4600 Cold-formed Steel Structures, Standards of Australian and Standards of New Zealand, Sydney-Wellington, Australia, 2005.

[16] AISI, North American Specifications for the Design of Cold-formed Steel Structural Members (NAS), Washington DC, USA, 2007.

[17] B.W. Schafer, Review: The Direct Strength Method of cold-formed steel member design, Journal of Constructional Steel Research 64 (2008) 766-778.

[18] Z.J. Li, B.W. Schafer, Buckling analysis of cold-formed steel members with general boundary conditions using CUFSM: conventional and constrained finite strip methods, Saint Louis, Missouri, USA, 2010.

[19] S.S. Cheng, Fire performance of cold-formed steel sections, PhD Thesis, Plymouth University, UK, 2014.

[20] S. Cheng, L.Y. Li, B. Kim, Buckling analysis of cold-formed steel channel-section beams at elevated temperatures, Journal of Constructional Steel Research 104 (2015) 74-80.

[21] G.M.E. Cooke, Thermal bowing in fire and how it affects building design, Hertfordshire: Fire Research Station, 1988.

[22] J.P. Papangelis, G.J. Hancock, Computer analysis of thin-walled structural members, Computers \& Structures 56 (1995) 157-176. 
[23] B.W. Schafer, Cold-formed steel behaviour and design: Analytical and numerical modelling of elements and members with longitudinal stiffeners, PhD Thesis, Cornell University, 1997.

[24] X. Chu, Z. Ye, R. Kettle, et al., Buckling behaviour of cold-formed channel sections under uniformly distributed loads, Thin-Walled Structures 43 (2005) 531-542.

[25] X.T. Chu, Failure analysis of cold-formed steel sections, Thesis, Aston University, Birmingham, 2004. 


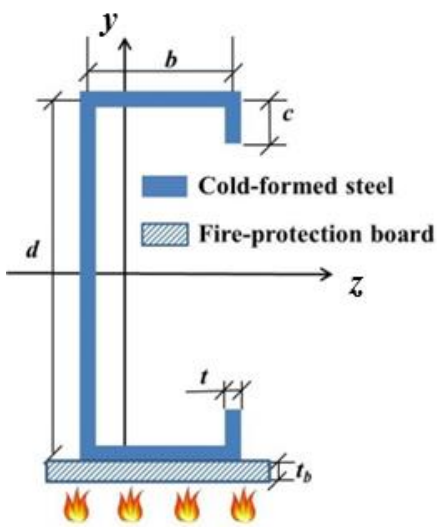

(a)

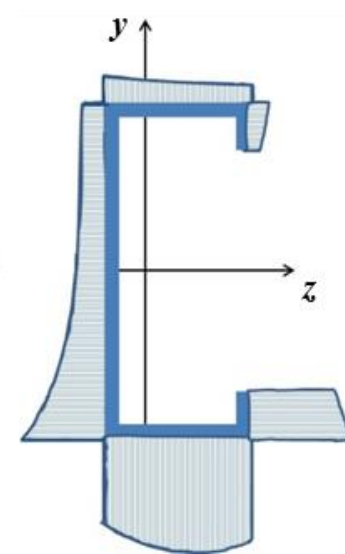

(b)

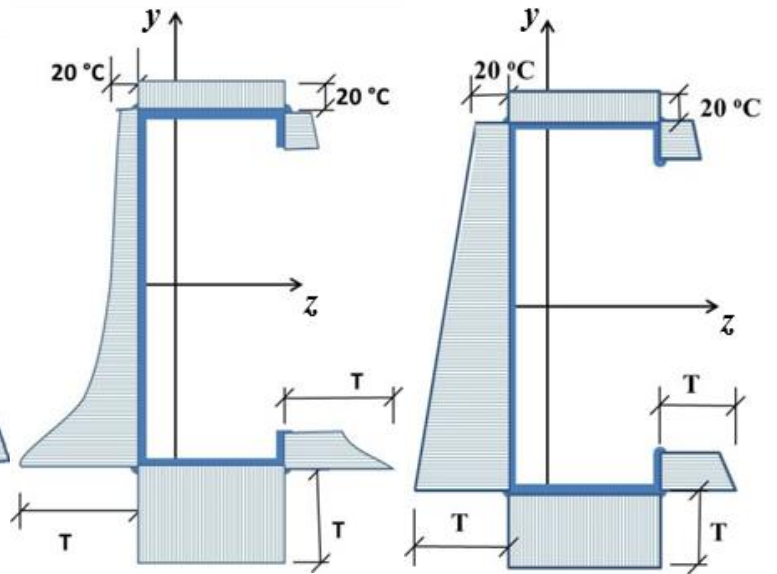

(c)

(d)

Figure 1. Temperature distributions of CFS channel-section columns in fire. (a) CFS sketch $\left(d=200 \mathrm{~mm}, b=75 \mathrm{~mm}, c=20 \mathrm{~mm}, t=2 \mathrm{~mm}, t_{b}=12.5 \mathrm{~mm}\right)$. (b) Temperature distribution calculated from FEA. (c) Assumed nonlinear temperature distribution. (d) Assumed linear temperature distribution. 


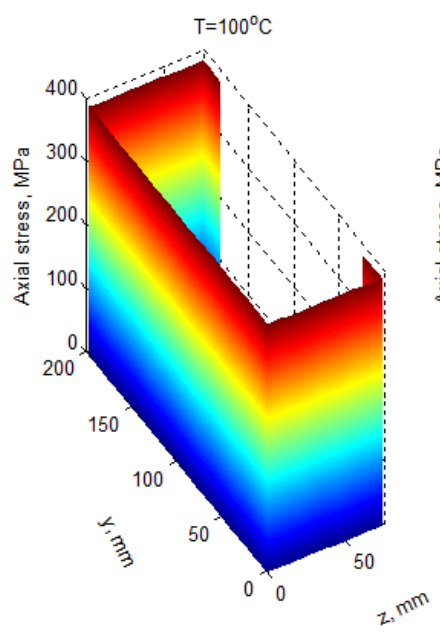

(a)

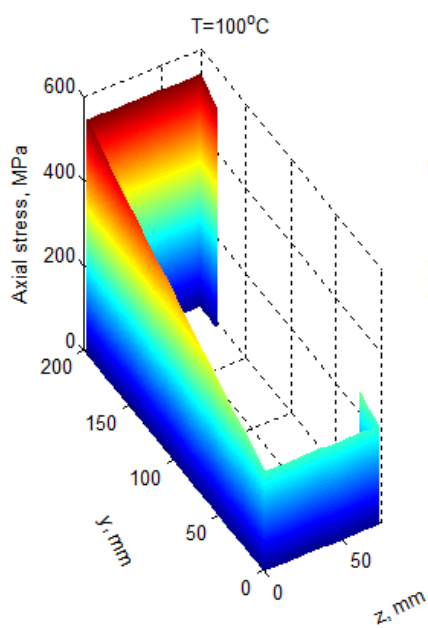

(d)

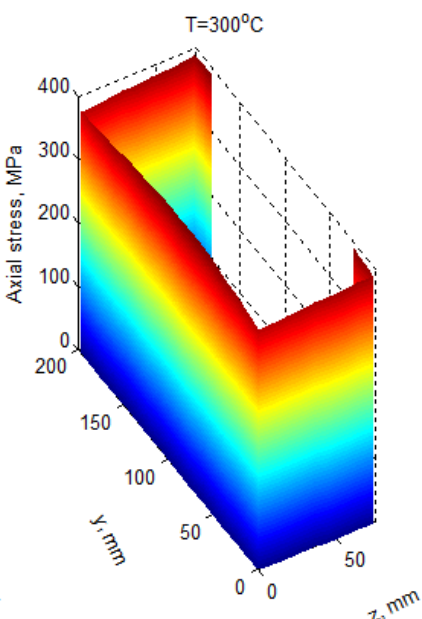

(b)
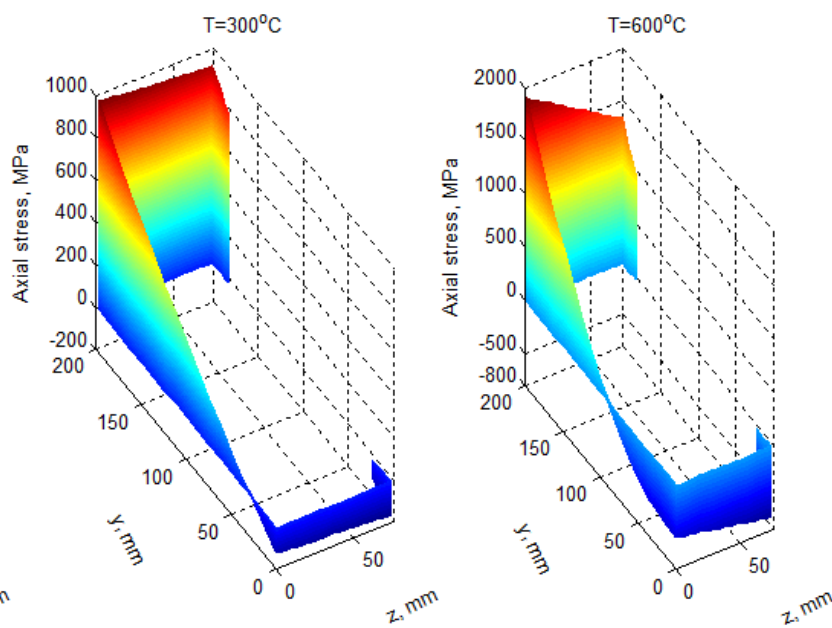

(f)

Figure 2. Pre-buckling stress distributions of a channel-section column under linear temperature distributions.

(a)-(c) Stress distribution at the end section of the column.

(d)-(f) Stress distribution at the middle section of the column. 


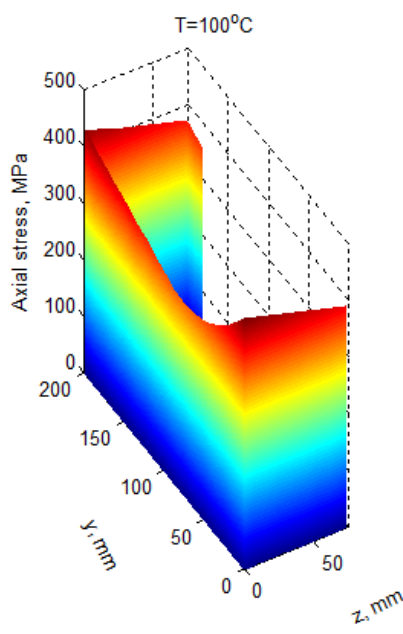

(a)

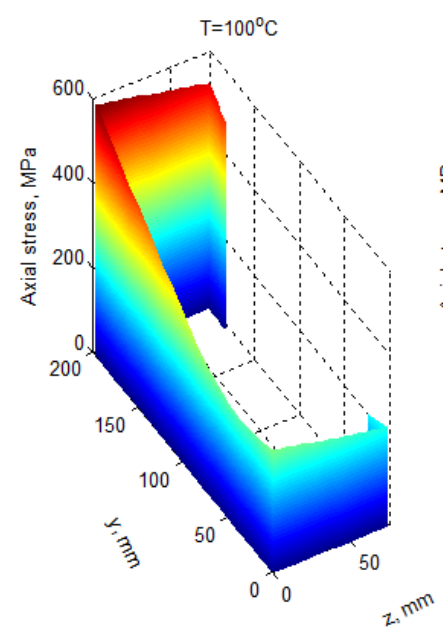

(d)

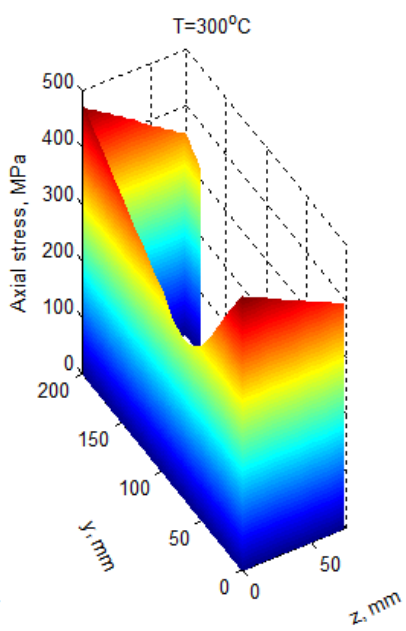

(b)

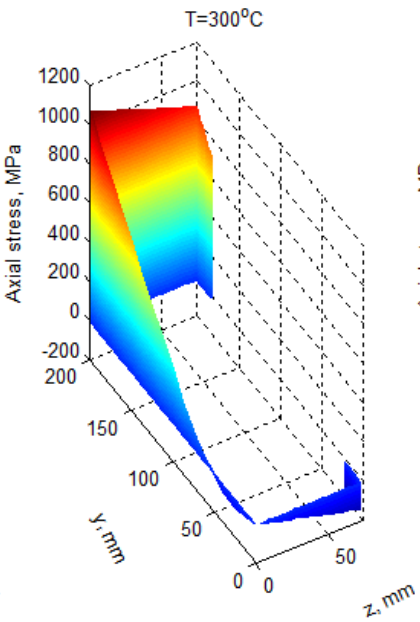

(e)

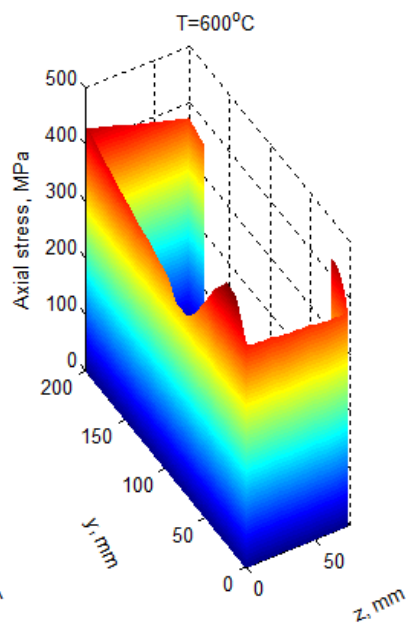

(c)

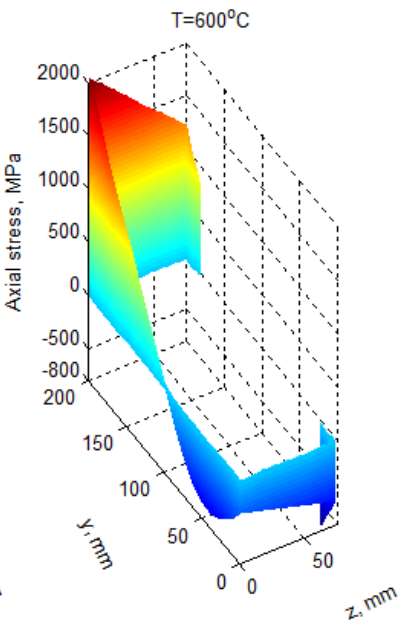

(f)

Figure 3. Pre-buckling stress distributions of a channel-section column under nonlinear temperature distributions.

(a)-(c) Stress distribution at the end section of the column.

(d)-(f) Stress distribution at the middle section of the column. 
Table 1 Comparison of elastic buckling loads of a CFS column under non-uniform temperature distributions with a maximum temperature of $300{ }^{\circ} \mathrm{C}$ (Unit: $\mathrm{kN}$ )

\begin{tabular}{|c|c|c|c|c|}
\hline \multirow{2}{*}{$\begin{array}{c}\text { Lengths } \\
(\mathrm{mm})\end{array}$} & \multicolumn{2}{|c|}{ Linear temperature distribution } & \multicolumn{2}{c|}{ Nonlinear temperature distribution } \\
\cline { 2 - 5 } & FEA & Modified FSM & FEA & Modified FSM \\
\hline 500 & 67.453 & 69.340 & 77.589 & 84.093 \\
\hline 1000 & 68.408 & 69.459 & 73.637 & 84.215 \\
\hline 2000 & 68.232 & 69.393 & 72.038 & 84.359 \\
\hline 3000 & 68.388 & 69.514 & 71.249 & 84.873 \\
\hline 4000 & 55.391 & 58.070 & 58.188 & 57.908 \\
\hline 5000 & 37.226 & 36.298 & 39.141 & 36.303 \\
\hline
\end{tabular}




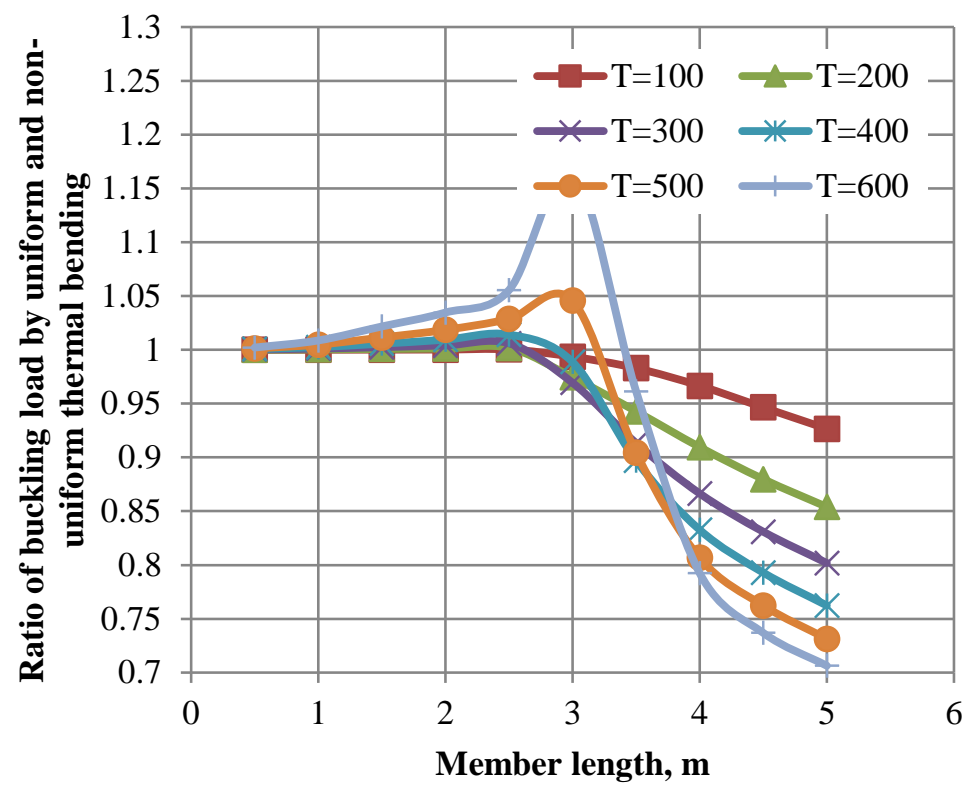

(a) Linear temperature distribution

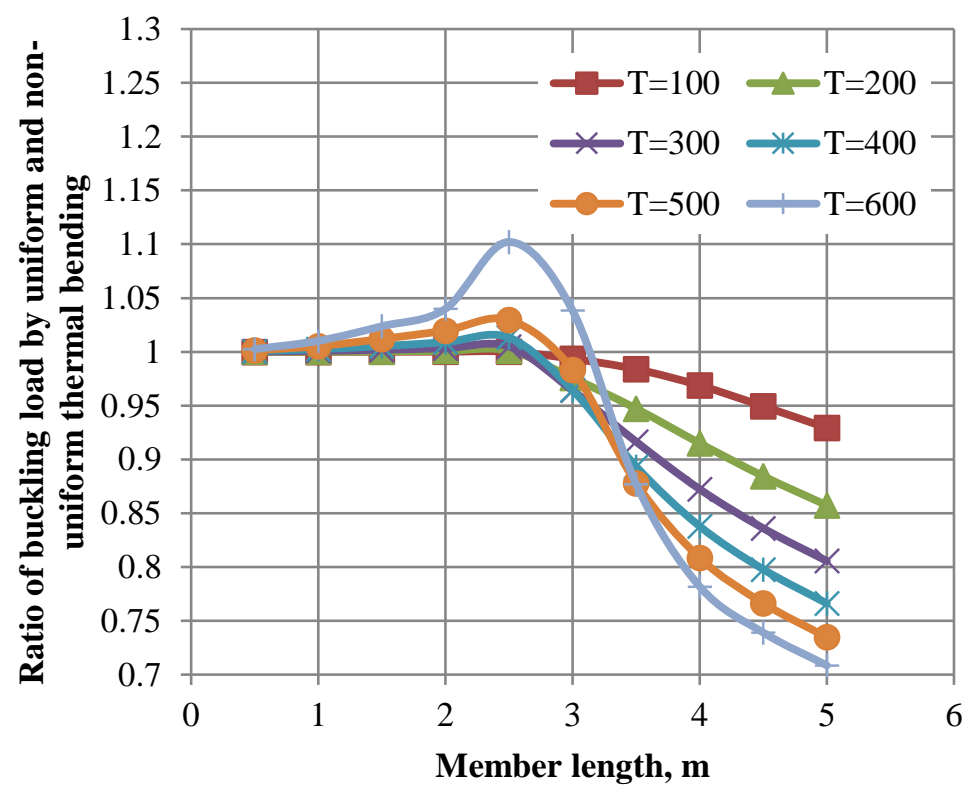

(b) Nonlinear temperature distribution

Figure 4 Comparison of elastic buckling loads of a CFS column calculated by using uniform and non-uniform thermal bending. 


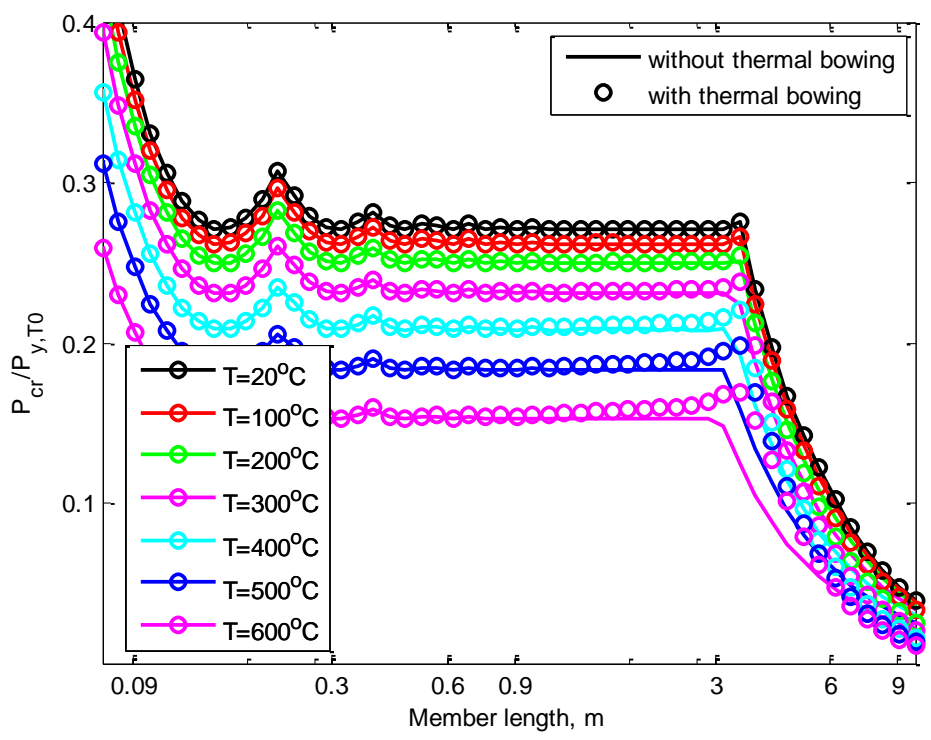

(a)

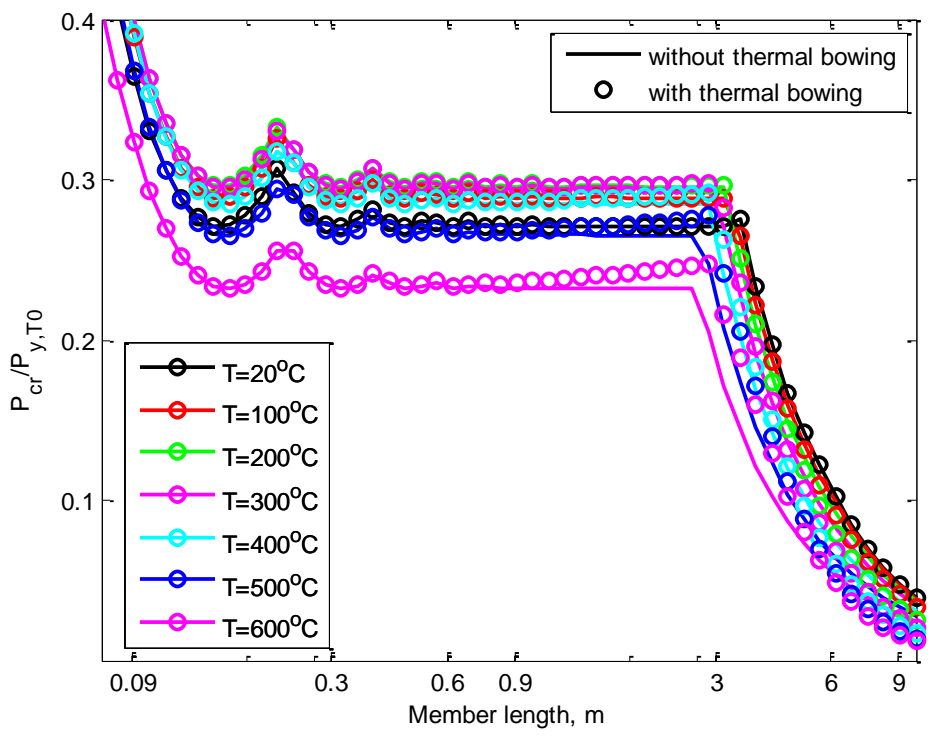

(b) 


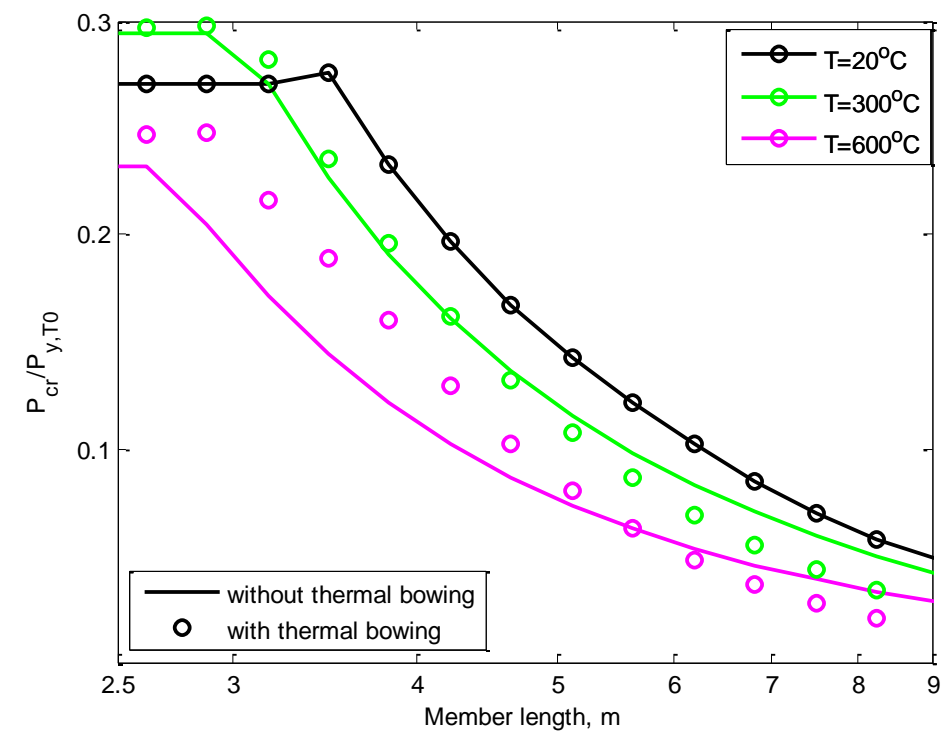

(c)

Figure 5. Critical buckling load $\left(P_{c r} / P_{y, T o}\right)$ of a channel-section column subjected to an axial load at various elevated temperatures. (a) Under linear temperature distributions. (b) Under nonlinear temperature distributions. (c) Global buckling curves under nonlinear temperature distributions. 


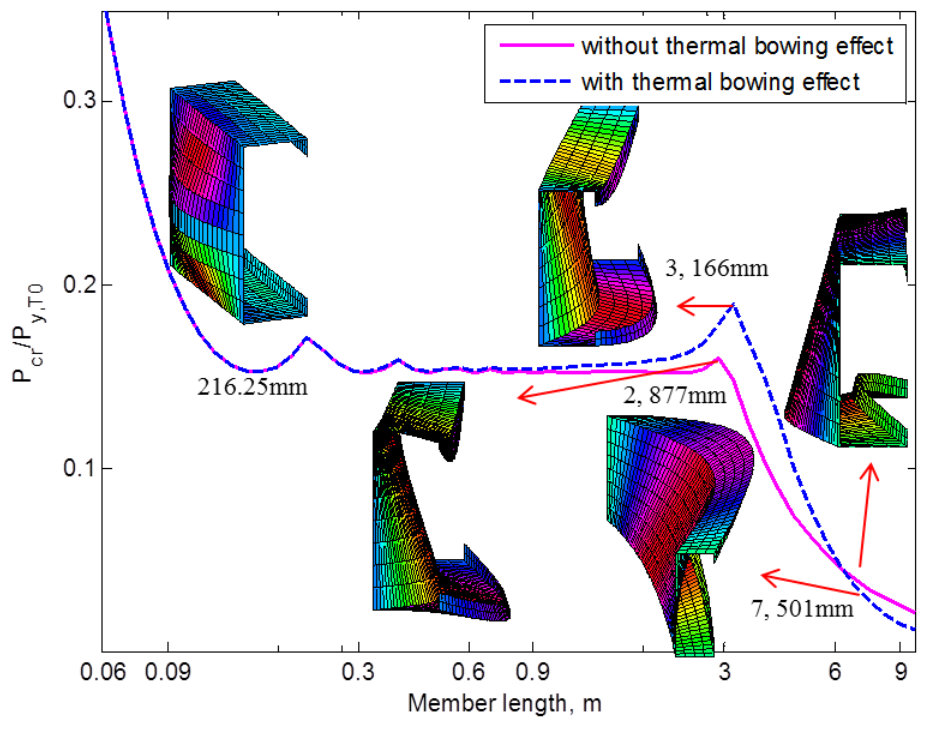

Fig. 6 Critical buckling modes of the CFS column under a linear temperature distribution when $\mathrm{T}=600{ }^{\circ} \mathrm{C}$. 


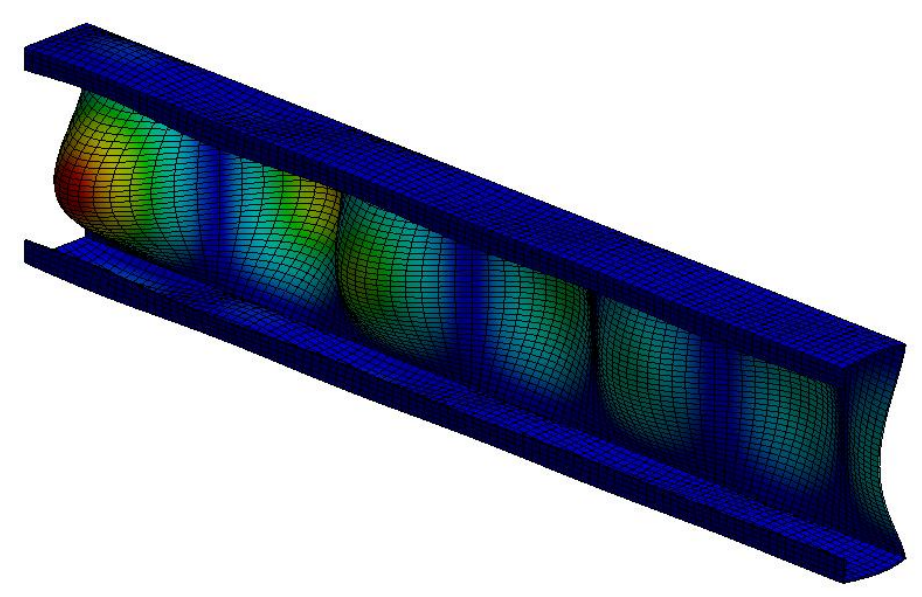

(a)

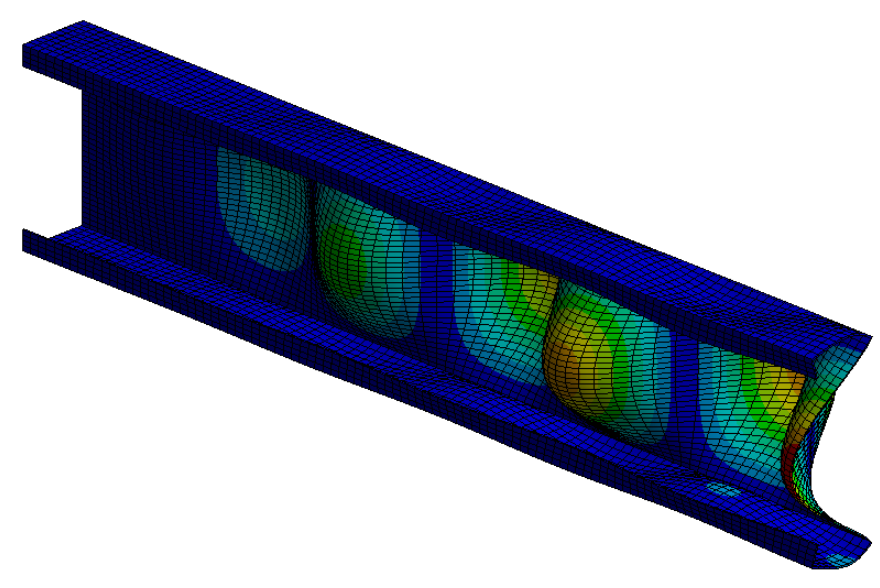

(b)

Fig. 7 Local buckling modes of a 2 m column under non-uniform temperature distributions.

(a) Linear temperature distribution. (b) Nonlinear temperature distribution 


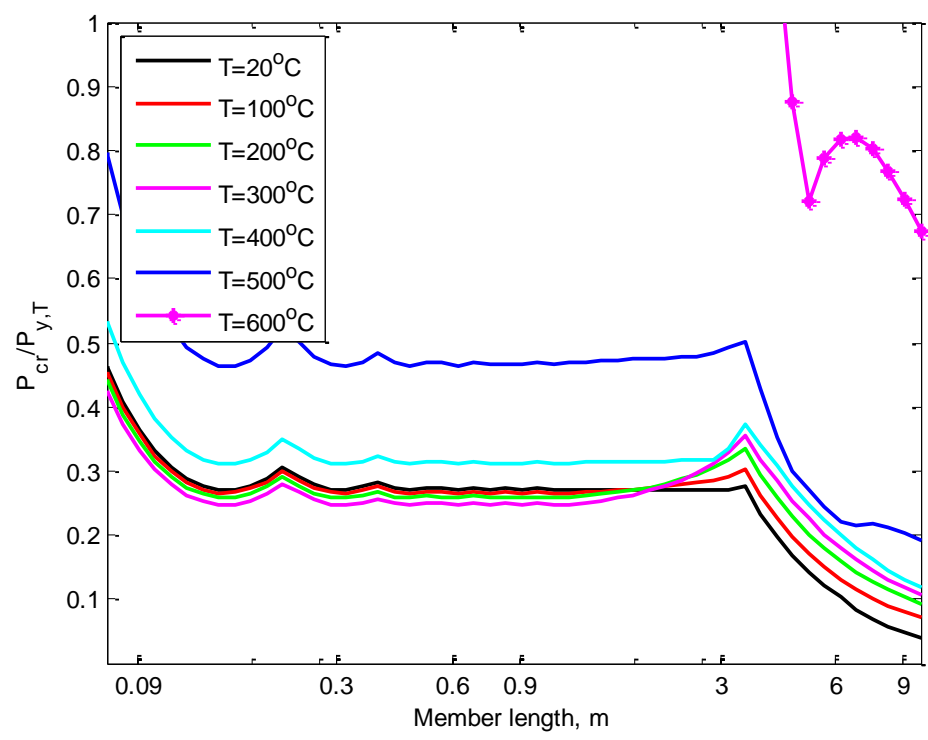

(a)

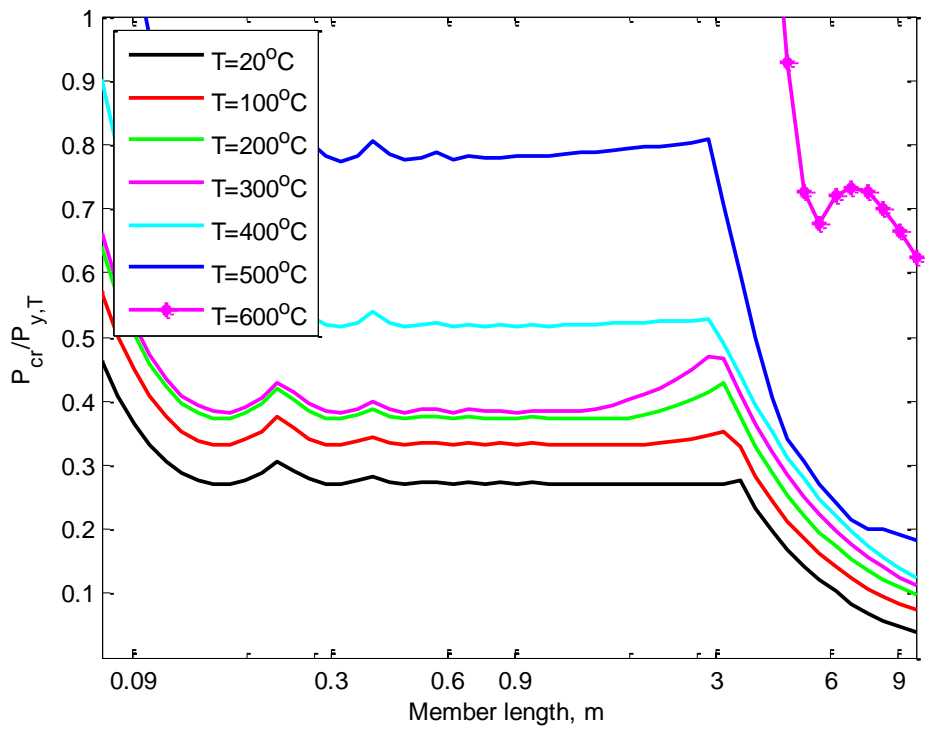

(b)

Figure 8. Relative critical load $\left(P_{c r} / P_{y, T}\right)$ of a channel-section column subjected to an axial load at various elevated temperatures. Section dimensions: $d=200 \mathrm{~mm}, b=75 \mathrm{~mm}, c=20$ $\mathrm{mm}, t=2 \mathrm{~mm}$. (a) Under linear temperature distributions. (b) Under nonlinear temperature distributions. 


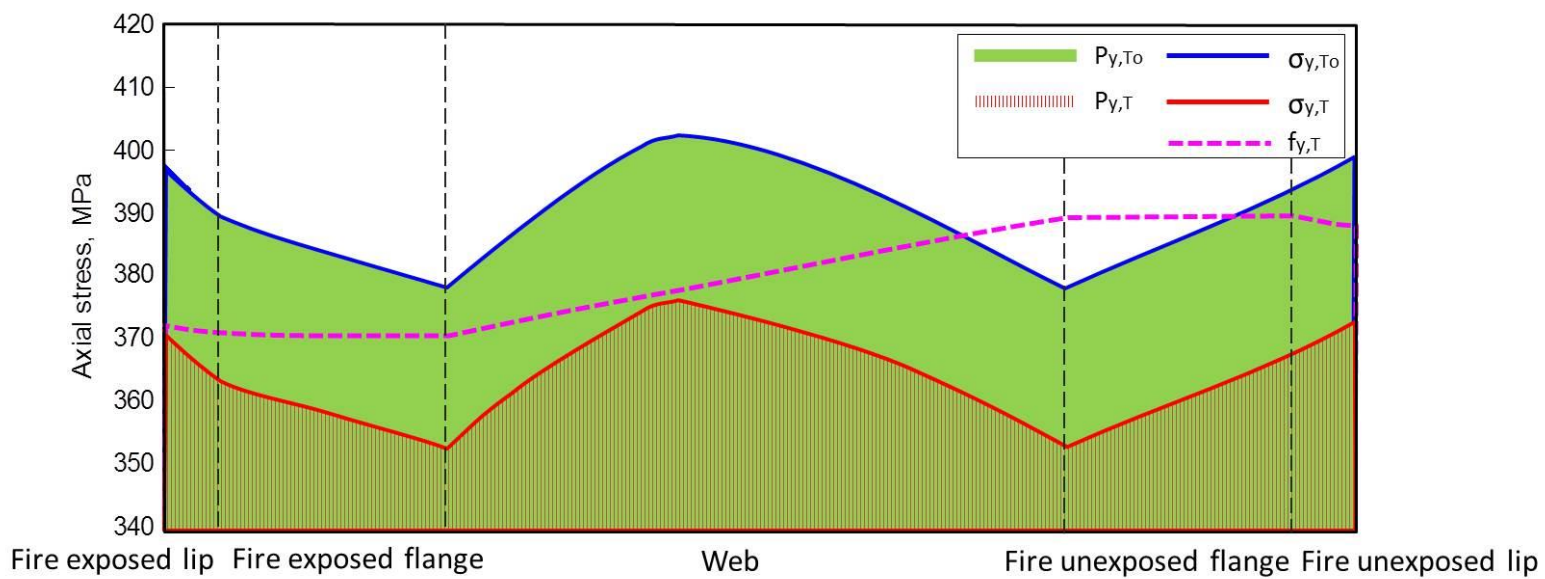

Figure 9. Relationship between $P_{y, T}$ and $P_{y, T o}$. under non-uniform temperatures. 